\title{
Theoretical Simulation and Optimization on Material Parameters of Thin Film Bulk Acoustic Resonator
}

\author{
Tao Zhang, ${ }^{1,2}$ Fujun Liang, ${ }^{1}$ Min Li, ${ }^{1}$ Shaorong Li, ${ }^{1}$ Huafeng Pang, ${ }^{1}$ Sufang Wang, \\ Huaze Zhu, ${ }^{1}$ Zhengxin Yan, ${ }^{1}$ and Shenggui Zhao ${ }^{1}$ \\ ${ }^{1}$ College of Science, Xian University of Science and Technology, Xian 710054, China \\ ${ }^{2}$ Laboratory of Modern Acoustics, Institute of Acoustics, Nanjing University, Nanjing 210093, China
}

Correspondence should be addressed to Tao Zhang; tzhang-psu@yahoo.com

Received 20 June 2015; Accepted 6 September 2015

Academic Editor: Jun Chen

Copyright (C) 2015 Tao Zhang et al. This is an open access article distributed under the Creative Commons Attribution License, which permits unrestricted use, distribution, and reproduction in any medium, provided the original work is properly cited.

\begin{abstract}
The resonance frequency, $f_{s}$, and the effective electromechanical coupling factor, $k_{\mathrm{eff}}^{2}$, of thin film bulk acoustic resonators (FBARs) are derived by transfer matrix method. The effects of thickness and density of electrode on $f_{s}$ and $k_{\text {eff }}^{2}$ with different piezoelectric layers are investigated by numerical calculation method. The results show that thickness and density of electrode affect $f_{s}$ obviously, especially in large thickness and density area. Moreover, the effects of thickness, density, and acoustic velocity of electrode on $k_{\text {eff }}^{2}$ of FBAR were also studied. The results show that there is a maximum $k_{\text {eff }}^{2}$ corresponding to the composition of thickness and density of electrode which is about $20 \%$ over the original electromechanical factor of piezoelectric film. $k_{\text {eff }}^{2}$ is in the direct proportion to the density $\rho_{e}$ and $v_{e}$ of electrode, respectively. The electrode thickness affects $k_{\text {eff }}^{2}$ small with high $v_{e}$; moreover, when $v_{e}$ is high enough, then $k_{\text {eff }}^{2}$ has almost nothing to do with $d_{e} \cdot k_{\text {eff }}^{2}$ always rises with electrode thickness first and then descends with its rising, and the thickness corresponding to the maximum $k_{\text {eff }}^{2}$ is different with different electrode, but it always locates in the special area. All above results indicate that the thickness, density, and acoustic velocity of electrode are so important that these results can be applied to design FBAR.
\end{abstract}

\section{Introduction}

The thin film bulk acoustic resonators (FBARs) are extensively applied for filters, resonators, and sensors, since they were first realized with the resonance frequency of $1.9 \mathrm{GHz}$ in 1999, such as MEMS, biosensors, and gas sensors [1-8]. It is a kind of important device in electronic equipment, and so many kinds of FBARs with high frequency and small dimension have been fabricated during recent ten years for the demand of the industry $[9,10]$. The FBARs are especially expected to be investigated and applied by many semiconductor companies such as Agilent, Philip, Murata, and TDK, because of their excellent merits.

Most of researchers focus on the fabrications and the applications of FBAR, and there also are a few researches about numerical simulation and optimization of FBAR. Chao et al. studied the electrode effects of FBAR by Butterworthvan Dyke (BVD) equivalent circuit [11], and Chen and
Wang calculated the effective electromechanical coupling coefficient of FBAR [12], and Zhang et al. applied resonant spectrum method to characterize piezoelectric films in FBAR [13]. Besides, our research group published the research about the electrode effect of FBAR [14], and Naumenko analyzed the propagation of acoustic wave in FBAR with Finite Element Method [15], and Kvasov and Tagantsev calculated the nonlinear electrostrictive coefficient with first principles [16]. All of above researches aim at the excellent FBAR performance because the resonance frequency, the effective coupling coefficient, and the accurate optimization for the design of FBAR are the key points for FBAR performance.

As a kind of bulk acoustic resonator (BAR), the figure of merit of an FBAR can be defined by $M=k_{\text {eff }}^{2} \cdot Q_{S} /\left(1-k_{\text {eff }}^{2}\right)$, and $Q_{S}$ is the resonance quality factor which is obviously controlled by the piezoelectric layer and electrode effect, and the special research has been done by our research group [14], and so we deeply discuss the material parameter 


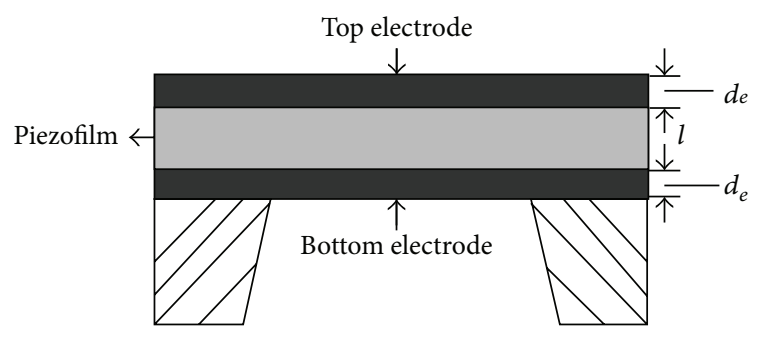

FIgURe 1: A typical FBAR structure.

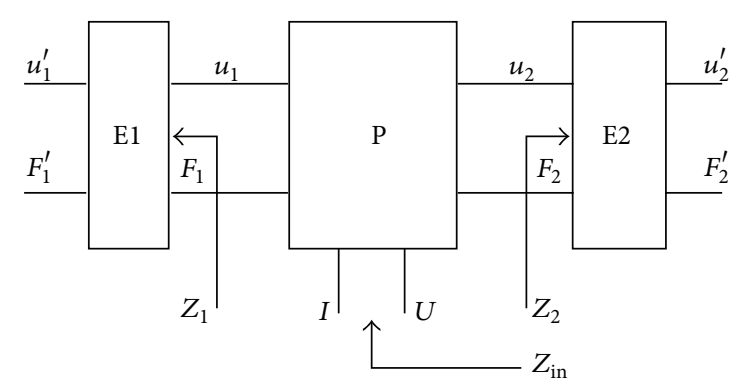

FIgURE 2: Transfer matrix schematic diagram.

effects of electrode on the resonance frequency, the effective electromechanical coupling factor, and the theoretical optimization consideration of FBAR in this paper considering the regularity among the density $\rho_{e}$, the thickness $d_{e}$, the acoustic velocity $v_{e}$ of electrode, and the FBAR performance parameters $f_{s}$ and $k_{\text {eff }}^{2}$. The transmission matrix method was used in this research, and the following research points and corresponding simulation results are presented: (1) the transfer matrix method being used to derive the input impedance equation, (2) effects of $\rho_{e}$ and $d_{e}$ on $f_{s}$, (3) effects of $\rho_{e}$ and $d_{e}$ on $k_{\text {eff }}^{2}$, (4) effects of $v_{e}$ and $d_{e}$ on $k_{\mathrm{eff}}^{2}$, and (5) the two cases with the piezoelectric films of $\mathrm{ZnO}$ and AlN, respectively, being compared for discussion.

\section{Simulation Method and Procedures}

For the FBAR shown in Figure 1, its transfer matrix schematic diagram is shown in Figure 2. P part represents the piezoelectric layer; E1 and E2 parts represent top electrode and bottom electrode, respectively. $U$ and $I$ represent electronic voltage and current port, respectively, $F$ and $u$ represent the force and vibration velocity, respectively, and $Z_{\text {in }}$ represents the input acoustic impedance of this system. So we can get the following transfer equation [13]:

$$
\left[\begin{array}{l}
U \\
I
\end{array}\right]=[B] \cdot\left[\begin{array}{l}
F \\
u
\end{array}\right]
$$

The piezoelectric transfer matrix $[B]$ can be given as [13]

$[B]$

$$
\begin{gathered}
=\frac{1}{\phi H}\left[\begin{array}{cr}
1 & \frac{j \phi^{2}}{\omega C_{0}} \\
j \omega C_{0} & 0
\end{array}\right] \\
\qquad\left[\begin{array}{cc}
\cos \gamma+j Z_{E} \sin \gamma & Z_{0}\left(Z_{E} \cos \gamma+j Z_{E} \sin \gamma\right) \\
\frac{j \sin \gamma}{Z_{0}} & 2(\cos \gamma-1)+j Z_{E} \sin \gamma
\end{array}\right],
\end{gathered}
$$

where $\phi=k_{t}^{2} C_{0} Z_{0} l / V$ is Mason equivalent circuit transfer ratio, $k_{t}^{2}$ is the electromechanical coupling factor of piezoelectric film, $C_{0}=\varepsilon_{33}^{S} S / l$ is the clamped capacitor with area of $S$, $\varepsilon_{33}^{S}$ is the dielectric constant with the vertical direction, $l$ is the thickness of piezoelectric film, $Z_{0}=\rho V S$ is the acoustic impedance of piezoelectric film with the density of $\rho, V$ is the longitudinal wave velocity, $\gamma=\omega l / V$ is the phase delay in the piezoelectric film, $\omega=2 \pi f$ is angular frequency, and $Z_{E}$ is the acoustic impedance of electrodes.

We take the electrode material as isotropic, and then we can get the matrix as

$$
\left[\begin{array}{l}
F_{1} \\
u_{1}
\end{array}\right]=\left[\begin{array}{cc}
\cos \gamma_{e 1} & j Z_{e 1} \sin \gamma_{e 1} \\
\frac{j \sin \gamma_{e 1}}{Z_{e 1}} & \cos \gamma_{e 1}
\end{array}\right] \cdot\left[\begin{array}{l}
F_{1}^{\prime} \\
u_{1}^{\prime}
\end{array}\right],
$$

where $F_{1}^{\prime}$ is zero because of the free top acoustic port, $\gamma_{e 1}=$ $\omega l_{e 1} / V_{e 1}$ is the phase delay in the top electrode, $l_{e 1}$ is the thickness of top electrode, $V_{e 1}$ is the acoustic velocity of top electrode, $Z_{e 1}=\rho_{e 1} V_{e 1} S$ is the acoustic impedance of top electrode, and $\rho_{e 1}$ is the density of top electrode.

We can get $Z_{1}$ from (3):

$$
Z_{1}=\frac{F_{0}}{u_{0}}=j Z_{e 1} \tan \gamma_{e 1} .
$$

Similarly, we can get the acoustic impedance of bottom electrode

$$
Z_{2}=\frac{F_{2}}{u_{2}}=j Z_{e 2} \tan \gamma_{e 2}
$$

So the total input acoustic impedance can be given as [13]

$$
\begin{aligned}
Z_{\text {in }} & =\frac{U}{I}=\frac{1}{j \omega C_{0}} \cdot\left[1-\frac{k_{t}^{2}}{\gamma}\right. \\
\cdot & \left.\frac{\left(z_{1}+z_{2}\right) \cdot \sin \gamma+j \cdot 2 \cdot(1-\cos \gamma)}{\left(z_{1}+z_{2}\right) \cdot \cos \gamma+j \cdot\left(1+z_{1} \cdot z_{2}\right) \cdot \sin \gamma}\right] .
\end{aligned}
$$

Inside (6), $z_{1}=Z_{1} / Z_{0}, z_{2}=Z_{2} / Z_{0}$. We take the top electrode and bottom electrode as the same, so the input impedance $\left(Z_{\text {in }}\right)$ can be given as $(7)$ with a transfer matrix method $[12,14]$

$$
Z_{\text {in }}=\frac{1}{j \omega C_{0}}\left[1-\frac{k_{t}^{2}}{\gamma} \frac{z \sin \gamma+j(1-\cos \gamma)}{z \cos \gamma+(j / 2)\left(1+z^{2}\right) \sin \gamma}\right] .
$$


$\gamma=\omega l / V$ is the phase shift in the piezofilm, and $z=Z / Z_{0}$ is the characteristic impedance ratio of the electrodes to the piezofilm as the top and bottom electrodes with the same material and thickness. The series resonance frequency $f_{s}$ and the parallel resonance frequency $f_{p}$ can be obtained by (7), and then the effective electromechanical coupling factor of FBAR can be demonstrated with the known $f_{s}$ and $f_{p}$ :

$$
k_{\text {eff }}^{2}=\frac{\left(f_{p}^{2}-f_{s}^{2}\right)}{f_{p}^{2}} \cong 2 \cdot \frac{\left(f_{p}-f_{s}\right)}{f_{p}} .
$$

Based on the definition published in the IEEE Std. 176-1987 [17], $f_{s}$ is the frequency corresponding with the maximum conductance. Then $f_{s}$ can be calculated by (7), and $k_{\text {eff }}^{2}$ can be evaluated by (8). However, $k_{\text {eff }}^{2}$ is not only determined by $k_{t}^{2}$ but also closely related with the electrode and resonator structure.

In the calculation of the resonance frequency $f_{s}$ with (7), the dielectric, the piezoelectric, and the elastic constants of the piezoelectric film and the elastic constants of the electrodes generally are complex values, but all others, such as $k_{t}^{2}$, are considered as real values [12]. In addition, for a given piezofilm, $C_{0}$ is a constant. The complex velocity $\widetilde{V}_{l}$ in piezofilms or electrodes was given by a complex expression [12]

$$
\widetilde{V}=\left[\frac{\left(C_{33}^{\prime}+j C_{33}^{\prime \prime}\right)}{\rho}\right]^{1 / 2} \cong V^{\prime}\left[1+\frac{j}{\left(2 Q_{m}\right)}\right] .
$$

$V^{\prime}=\sqrt{C_{33}^{\prime} / \rho}$ is the real part, $V^{\prime \prime}=\sqrt{C_{33}^{\prime \prime} / \rho}$ is the imaginary part, and $C_{33}^{\prime}$ and $C_{33}^{\prime \prime}$ are the real part and imaginary part of elastic constant $C_{33}$, where $C_{33}^{\prime \prime}$ is responsible for the mechanical losses, and $Q_{m}=C^{\prime} / C^{\prime \prime} \cong V^{\prime} / 2 V^{\prime \prime}$ is the mechanical quality factor of the piezofilm or the electrode.

So the resonance frequency and the effective electromechanical coupling coefficient of FBAR can be obtained and analyzed by above definitions and equations.

\section{Effects of Material Quality Factors on Effective Coupling Factor}

The effect of electrode on FBAR performance is studied and shown in the following parts, and the thickness, $d_{e}$, the density, $\rho_{e}$, on the resonance frequency, $f_{s}$, and the effective electromechanical coupling factor, $k_{\text {eff }}^{2}$, are investigated; besides, the acoustic velocity $v_{e}$ on $k_{\text {eff }}^{2}$ is also done. The different piezoelectric films of $\mathrm{ZnO}$ and $\mathrm{AlN}$ with thickness of $2 \mu \mathrm{m}$ are used in this research, and they both are typical examples in the realistic applications, and the constants of material used in the calculation are shown in Table 1.

3.1. Effects of $\rho_{e}$ and $d_{e}$ on $f_{s}$ of FBAR. The resonance frequency is very important parameter for frequency devices, especially for the acoustic device with vibration such as FBAR. In general, the frequency of FBAR is the basic parameter which should be considered during the design of FBAR, and so it is why we began with it.
TABLE 1: Data of material properties.

\begin{tabular}{lcccc}
\hline Material & $\begin{array}{c}\text { Density } \\
\left(\mathrm{kg} / \mathrm{m}^{3}\right)\end{array}$ & $\begin{array}{c}\text { Velocity } \\
(\mathrm{m} / \mathrm{s})\end{array}$ & $\begin{array}{c}\text { Impedance } \\
\left(10^{6} \mathrm{~kg} / \mathrm{m}^{3} \mathrm{~s}\right)\end{array}$ & $k_{t}^{2}$ \\
\hline $\mathrm{ZnO}$ & 5606 & 6350 & 35.6 & 7.50 \\
$\mathrm{AlN}$ & 3300 & 11050 & 36.5 & 6.25 \\
\hline
\end{tabular}

The resonance frequency definitions are stated in the IEEE Std. 176-1987 that $f_{s}$ is defined as the frequency of maximum conductance [17]. So we can calculate $f_{s}$ by setting the conductance maximum with (7). In the calculations, we take the different piezoelectric films with $\mathrm{ZnO}$ and $\mathrm{AlN}$ and take all other parameters as constants except the density and the thickness of electrode. We can take the different density as different electrode material and study what will happen to the resonance frequency of FBAR if we change the thickness of the same material electrode, which is very valuable for the design of FBAR devices.

The results of $f_{s}$ changing with $\rho_{e}$ and $d_{e}$ of FBAR based on $\mathrm{ZnO}$ and AlN piezoelectric films were obtained, and the three-dimensional (3D) figures were shown as Figures $3(\mathrm{a})$ and 4(a), respectively, and the two-dimensional (2D) figures were shown as Figures 3(b) and 4(b), respectively. It can be obtained that the resonance frequency decreases with the thickness and the density of electrode increasing by the 3D figures, and this rule becomes more typical with the thin electrode or the high density, and it is the most obvious when both thickness and density of electrode appear in large value area. So we can choose proper material with small density and thin electrode for high resonance frequency by these results even though there still are some other parameters which should also be considered such as acoustic velocity. However, if the acoustic velocity in the electrode is a constant, then this result can be effectively used to design and evaluate the resonance frequency according to the application requirement. The above results can also be proved by the two 2D figures. Besides, we also can get that the FBAR decreased over half of resonance frequency just with the thickness change of $0.4 \mu \mathrm{m}$, and so the thickness effect of electrode on the resonance frequency are very distinct, which becomes smoother in the smaller thickness area.

3.2. Effects of $\rho_{e}$ and $d_{e}$ on $k_{e f f}^{2}$ of FBAR. Based on the definition published in the IEEE Std. 176-1987 [17], $f_{s}$ is the frequency of maximum conductance and $f_{p}$ is the frequency of maximum resistance. Then $f_{s}$ and $f_{p}$ can be calculated by (7), and $k_{\text {eff }}^{2}$ can be evaluated by (8). $k_{\text {eff }}^{2}$ is not only determined by $k_{t}^{2}$ but also determined by the electrode and resonator structure.

We calculated $k_{\text {eff }}^{2}$ changing with the thickness and the density of electrode, and the 3D figures and 2D figures were given in Figures 5 and 6 with $\mathrm{ZnO}$ and $\mathrm{AlN}$ piezoelectric films, respectively.

It can be obtained by Figures 5(a) and 6(a) that there is the maximum $k_{\text {eff }}^{2}$ corresponding to some compositions of thickness and density of electrode, which is near the thin electrode and high density area. $k_{\text {eff }}^{2}$ increases and then 

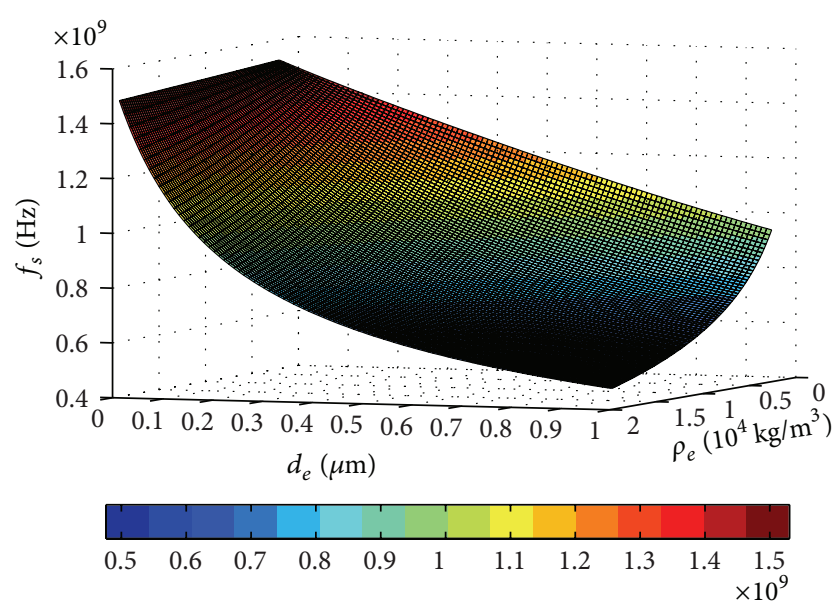

(a)

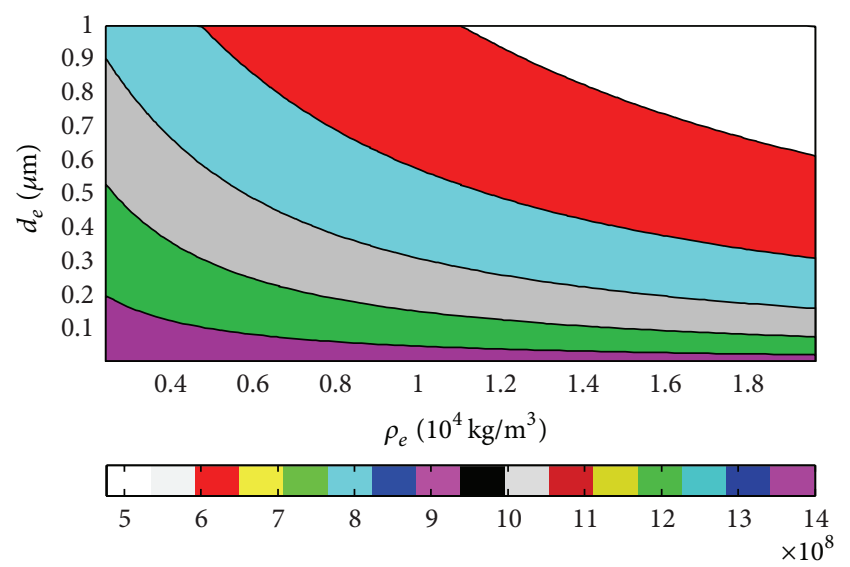

(b)

FIgURE 3: $f_{s}$ of FBAR versus the thickness and the density of electrode with $\mathrm{ZnO}$ piezoelectric film.

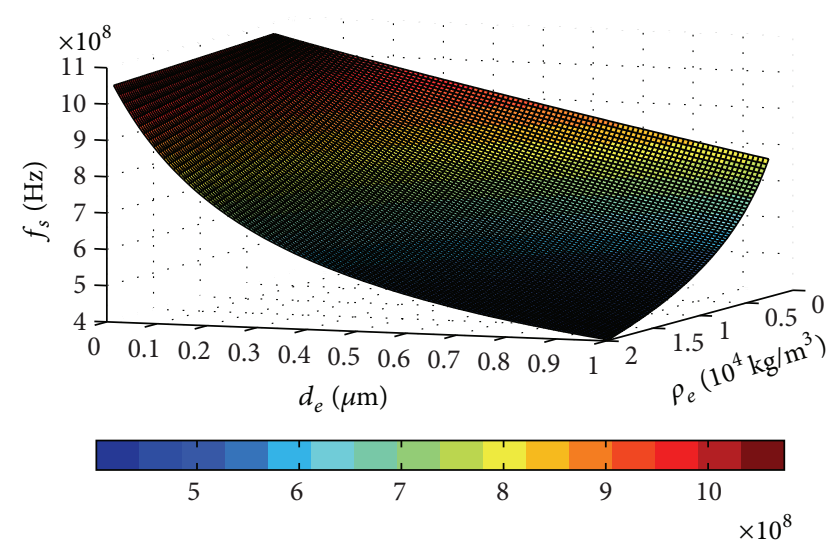

(a)

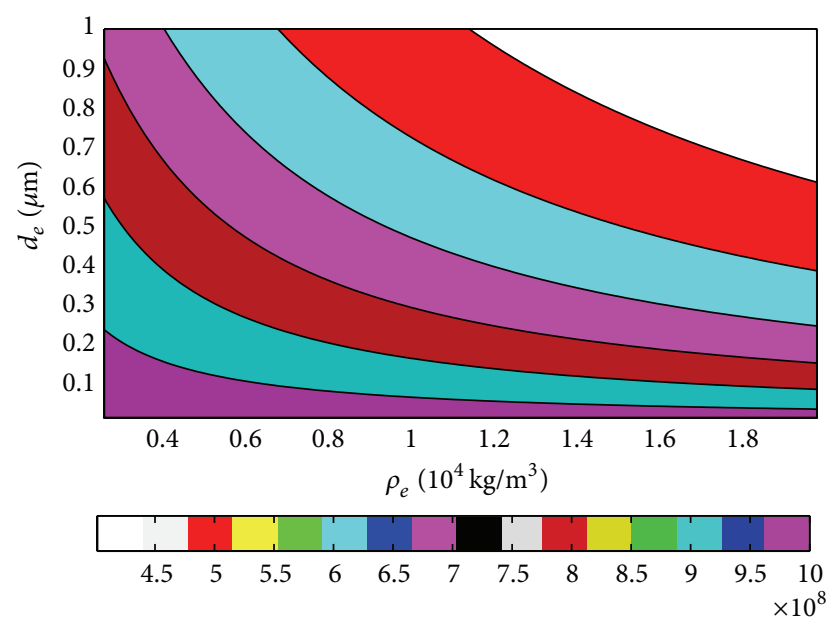

(b)

FIGURE 4: $f_{s}$ of FBAR versus the thickness and the density of electrode with AlN piezoelectric film.

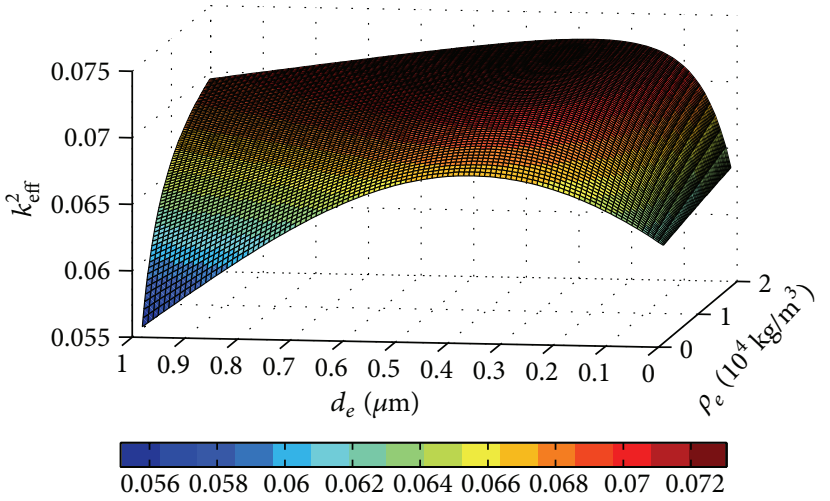

(a)

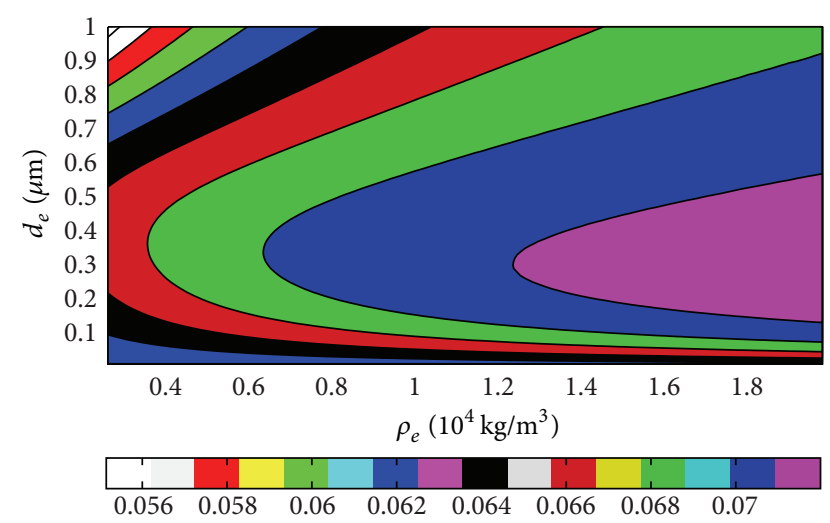

(b)

FIGURE 5: $k_{\text {eff }}^{2}$ of FBAR versus the thickness and the density of electrode with $\mathrm{ZnO}$ piezoelectric film. 


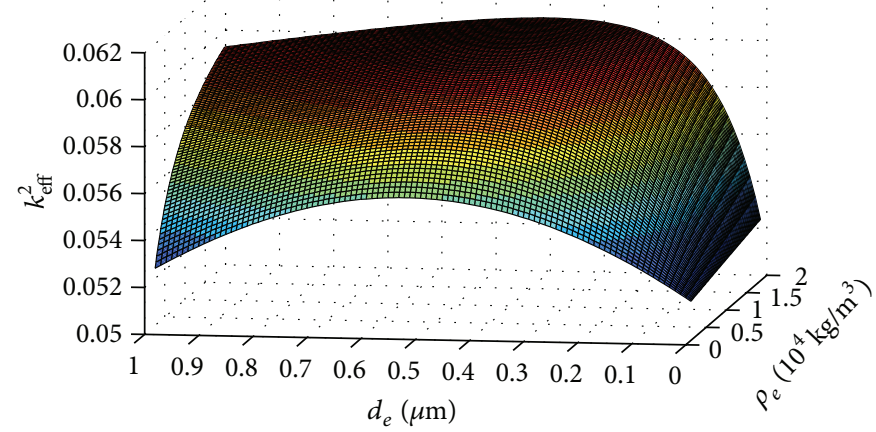

$\begin{array}{llllllllll}0.052 & 0.053 & 0.054 & 0.055 & 0.056 & 0.057 & 0.058 & 0.059 & 0.06\end{array}$

(a)

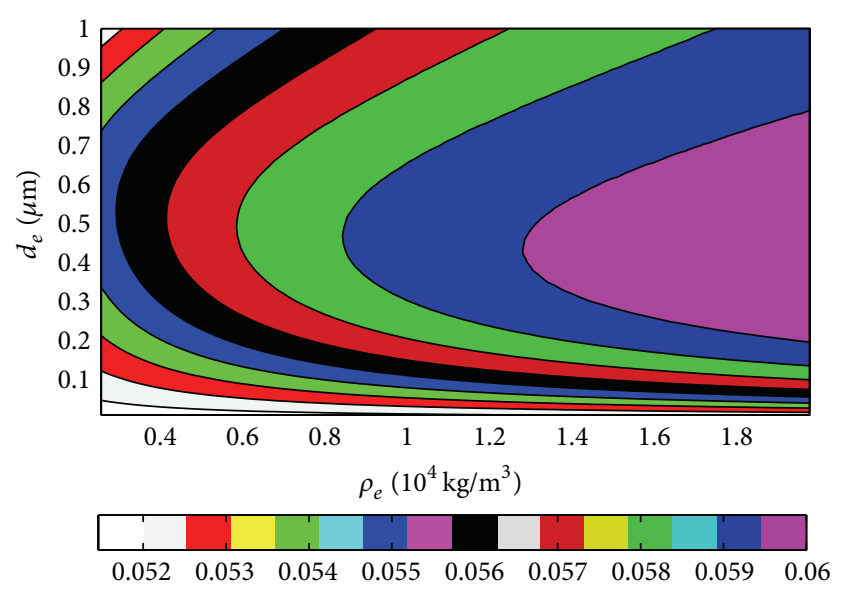

(b)

FIGURE 6: $k_{\text {eff }}^{2}$ of FBAR versus the thickness and the density of electrode with AlN piezoelectric film.

decreases with the thickness increasing, and the increasing ratio can reach to $20 \%$ over original value of piezoelectric film. However, $k_{\text {eff }}^{2}$ mostly increases with the density of electrode. $k_{\text {eff }}^{2}$ changes fast with the thickness of electrode when the density of electrode is small, and vice versa. The FBAR with the $\mathrm{ZnO}$ layer shows that $k_{\text {eff }}^{2}$ changes more quickly than the case of AlN, and the thickness effect is more obvious too, especially for the small density of electrode cases. Furthermore, we can also get the same witness from the $2 \mathrm{D}$ figures of Figures 5(b) and 6(b).

A very interesting result is obtained from $2 \mathrm{D}$ figures that $k_{\text {eff }}^{2}$ will arrive at a maximum area with special thickness values for any density of electrode, and the corresponding thickness area is very obvious at the center, such as the FBAR with $\mathrm{ZnO}$. The thickness beginning with the maximum $k_{\text {eff }}^{2}$ area is nearby $0.37 \mu \mathrm{m}$, and the thickness is nearby $0.5 \mu \mathrm{m}$ for the AlN case. This is a very interesting and important result, and it means the effect of thickness on $k_{\text {eff }}^{2}$ has a strong rule which is affected little by the density of electrode material. We analyzed this phenomenon that it is the thickness where standing wave appears, and the difference between these two piezoelectric film cases should be attributed to the different piezoelectric thin films. Moreover, it confirms that the standing wave exists in the FBAR, and the thickness of electrode should be optimized with this merit point, which is effective for all kinds of electrode materials.

3.3. Effects of $v_{e}$ and $d_{e}$ on $k_{e f f}^{2}$ of FBAR. For overall evaluation of $k_{\text {eff }}^{2}$, the effects of $v_{e}$ and $d_{e}$ on $k_{\text {eff }}^{2}$ were conducted with the $2 \mu \mathrm{m}$ thick piezoelectric film, and the $2 \mathrm{D}$ and $3 \mathrm{D}$ figures with four different electrode density choices were given for contrasting. The $3 \mathrm{D} k_{\mathrm{eff}}^{2}$ versus $v_{e}$ and $d_{e}$ curves based on $\mathrm{ZnO}$ and $\mathrm{AlN}$ were shown in Figures 7 and 9, respectively, and the $2 \mathrm{D}$ results were shown in Figures 8 and 10 correspondingly. The key parameters and results were marked on the 3D figures. We can get that $k_{\text {eff }}^{2}$ always rises with the density of electrode for the $\mathrm{ZnO}$ case in Figure 7

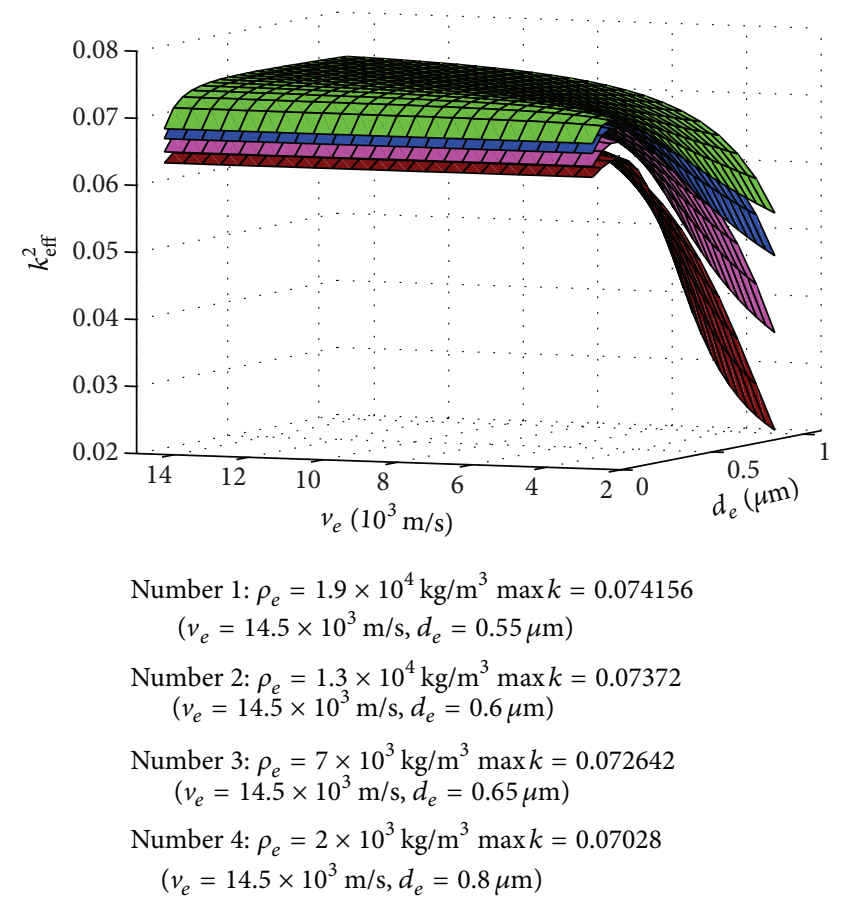

FIGURE 7: $k_{\text {eff }}^{2}$ of FBAR versus the thickness and the acoustic velocity of electrode with $\mathrm{ZnO}$ piezoelectric film and different density (3D curves).

which also can be observed in above section results, and the maximum $k_{\text {eff }}^{2}$ always appears at the maximum acoustic velocity during our calculation range. The AlN case is the same behavior as the $\mathrm{ZnO}$ FBAR in Figure 9. Moreover, $k_{\mathrm{eff}}^{2}$ rises more obviously with $v_{e}$ in the large electrode thickness area and vice versa, and $k_{\text {eff }}^{2}$ changes little with $v_{e}$ among the small electrode thickness area and especially least in the high density electrode material area. On the other hand, $k_{\text {eff }}^{2}$ rises distinctly with the electrode thickness in the low velocity area, but it almost does not change in the high velocity space. 


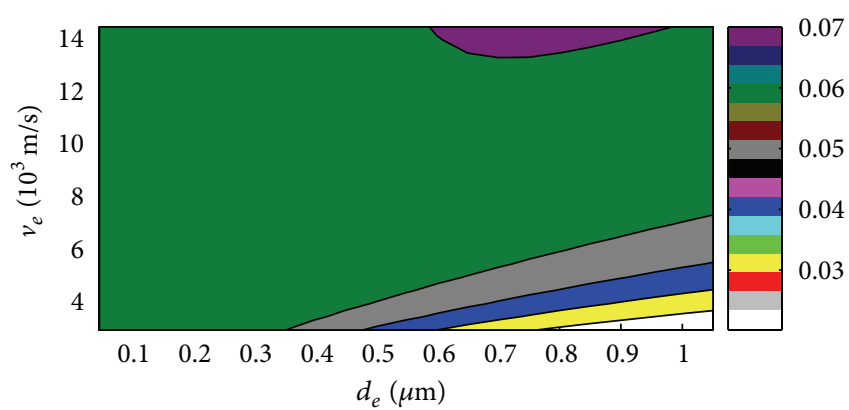

(a) $\rho_{e}=2 \times 10^{3} \mathrm{~kg} / \mathrm{m}^{3}$

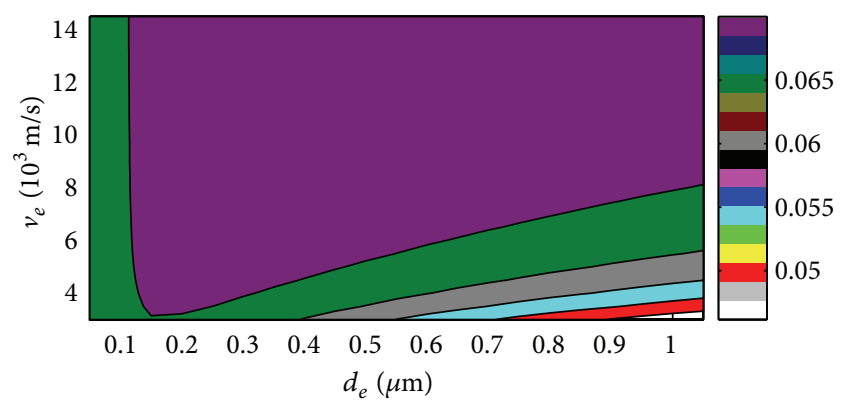

(c) $\rho_{e}=1.3 \times 10^{4} \mathrm{~kg} / \mathrm{m}^{3}$

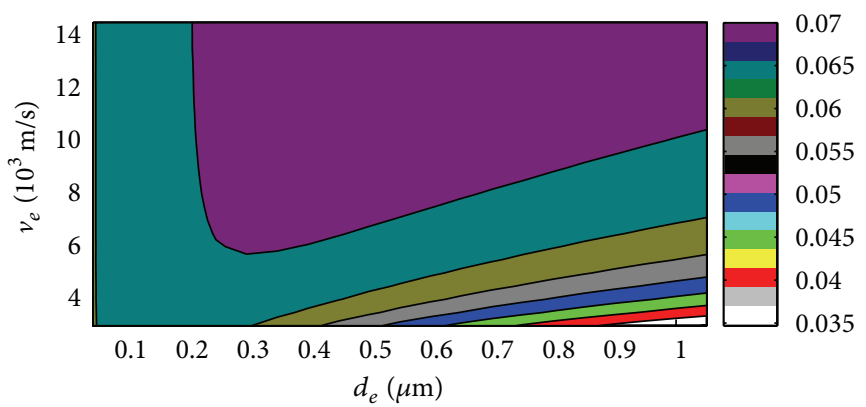

(b) $\rho_{e}=7 \times 10^{3} \mathrm{~kg} / \mathrm{m}^{3}$

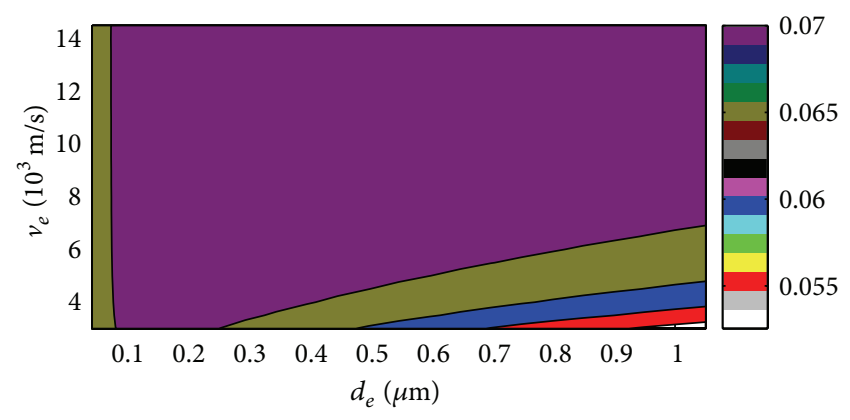

(d) $\rho_{e}=1.9 \times 10^{4} \mathrm{~kg} / \mathrm{m}^{3}$

FIGURE 8: $k_{\text {eff }}^{2}$ of FBAR versus the thickness and the acoustic velocity of electrode with ZnO piezoelectric film (2D curves).

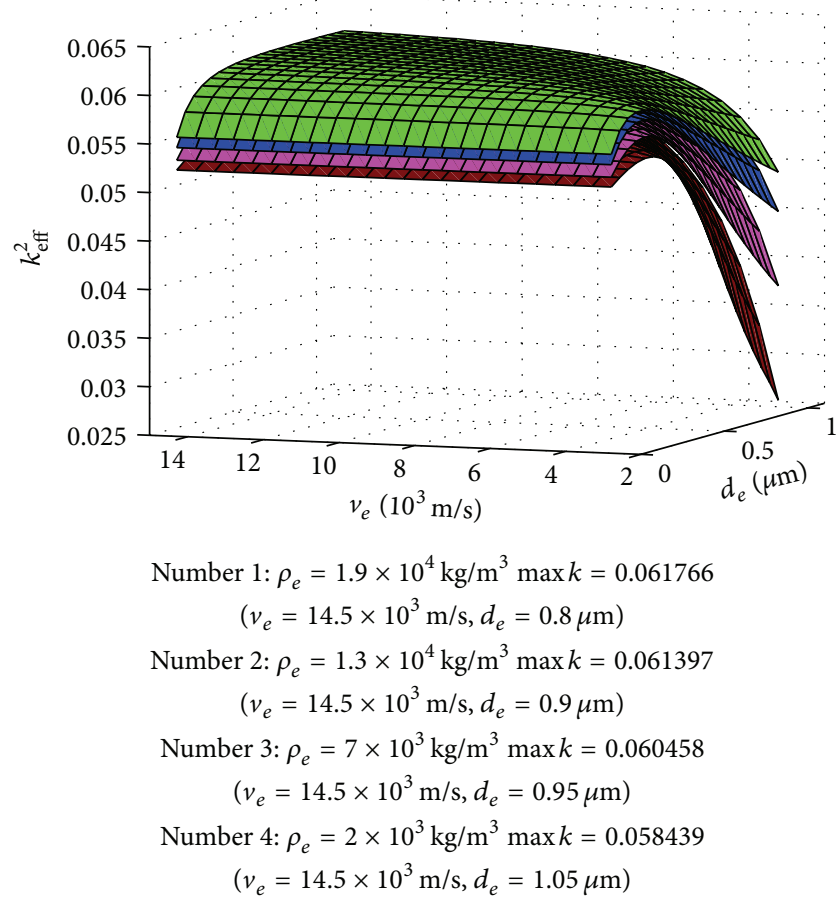

FIGURE 9: $k_{\text {eff }}^{2}$ of FBAR versus the thickness and the acoustic velocity of electrode with AlN piezoelectric film and different density (3D curves).

We can conclude that $k_{\text {eff }}^{2}$ is in the direct proportion to the density $\rho_{e}$ here, and the acoustic velocity of electrode has a similar effect too, and $k_{\text {eff }}^{2}$ dominantly rises with $v_{e}$. The electrode thickness affects small $k_{\text {eff }}^{2}$ with high $v_{e}$; moreover, when $v_{e}$ is high enough, then $k_{\text {eff }}^{2}$ has almost nothing to do with $d_{e}$. But $k_{\text {eff }}^{2}$ changes fast with $d_{e}$ when $v_{e}$ is small, and $k_{\text {eff }}^{2}$ rises with electrode thickness first and then descends with its rising, which was also observed in the former section. The thickness corresponding to the maximum $k_{\text {eff }}^{2}$ is different with different electrode density; however, it descends with density ascending, which also proves the special value $\rho_{e} d_{e}$ corresponding with the maximum $k_{\text {eff }}^{2}$ of FBAR even though we cannot get this result directly by these figures because there are not so many piezoelectric film parameters considered for proving [14]. All these behaviors are similar for both $\mathrm{ZnO}$ and AlN based FBARs.

The density, acoustic velocity, and thickness of electrode of FBAR can be optimized by above results for special $f_{s}$ and $k_{\text {eff }}^{2}$ need. We can get the higher $k_{\text {eff }}^{2}$ with the higher electrode density, and it is also the same to acoustic velocity of electrode material. The best thickness of electrode is decided by density and especially acoustic velocity; however, the thickness effects are obvious when the acoustic velocity is small, but it almost does not change if the acoustic velocity is very big. Moreover, the best thickness corresponding to the maximum $k_{\text {eff }}^{2}$ is different with different cases, but the values are in the special area comparing with the distinctly variable $v_{e}$ and $\rho_{e}$, and it is because we take most of the parameters of piezoelectric thin film as constants, even though these results can be observed in both $\mathrm{ZnO}$ and AlN FBARs in this research.

\section{Conclusions}

In this paper, the following research and results have been given. 


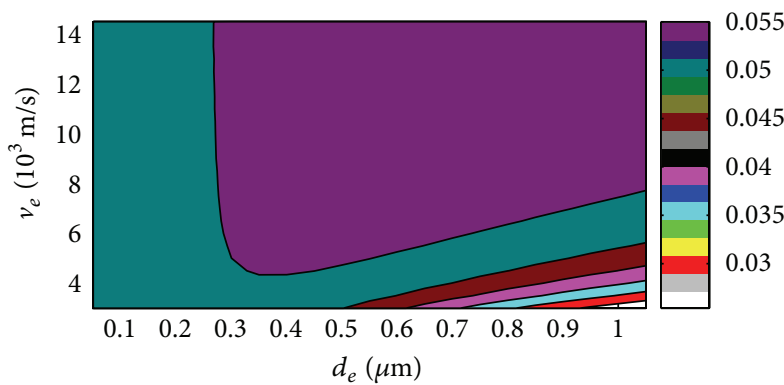

(a) $\rho_{e}=2 \times 10^{3} \mathrm{~kg} / \mathrm{m}^{3}$

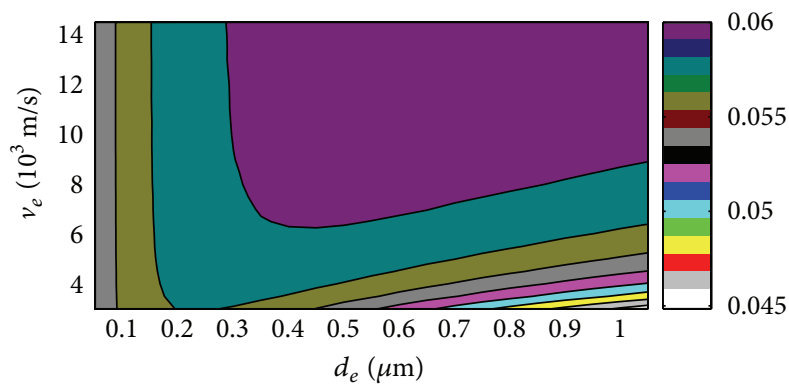

(c) $\rho_{e}=1.3 \times 10^{4} \mathrm{~kg} / \mathrm{m}^{3}$

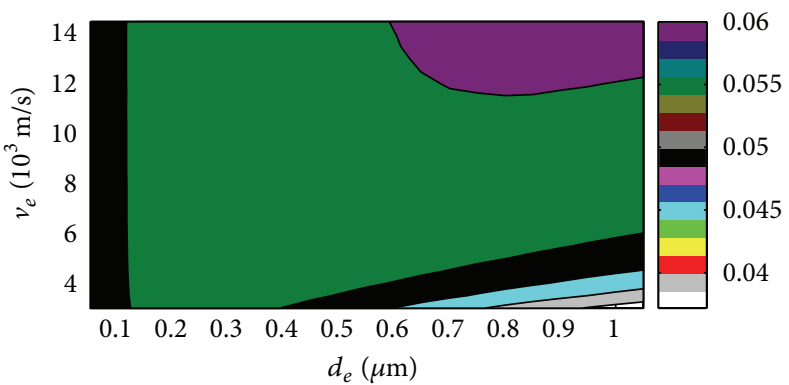

(b) $\rho_{e}=7 \times 10^{3} \mathrm{~kg} / \mathrm{m}^{3}$

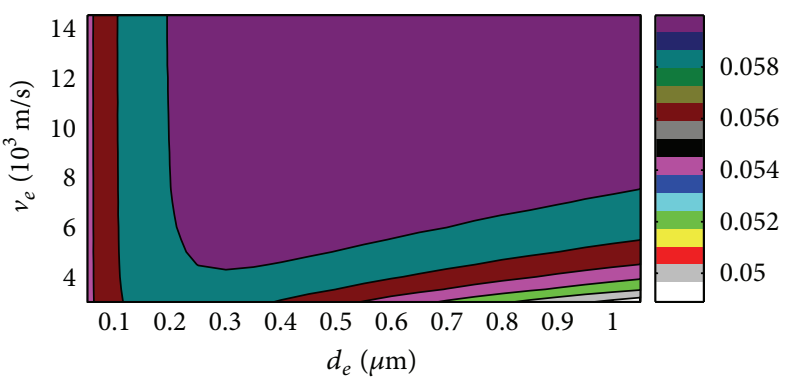

(d) $\rho_{e}=1.9 \times 10^{4} \mathrm{~kg} / \mathrm{m}^{3}$

FIGURE 10: $k_{\text {eff }}^{2}$ of FBAR versus the thickness and the acoustic velocity of electrode with AlN piezoelectric film and different density (2D curves).

(i) Firstly, we derived the input acoustic impedance equation with transfer matrix method, and the simplified impedance equation of FBAR with the same top and bottom electrode is obtained. This equation can be applied extensively to calculate the resonance frequency, the effective electromechanical coupling factor, the mechanical quality factor, and the merit figure of FBAR, which makes the theoretical calculation and optimization easy.

(ii) Secondly, the effect of thickness and density of electrode on the resonance frequency of FBAR was investigated, and it can be obtained that the thickness and the density of electrode affect the resonance frequency obviously, especially at the large thickness and density area. This result shows that the thickness and the material of electrode are very key parameters for the resonance frequency design of FBAR.

(iii) Finally, the effects of thickness, density, and acoustic velocity on the effective electromechanical coupling factor were studied, respectively. The results show that $k_{\mathrm{eff}}^{2}$ is in the direct proportion to both density $\rho_{e}$ and $v_{e}$ of electrode. The electrode thickness affects small $k_{\text {eff }}^{2}$ with high $v_{e}$; moreover, when $v_{e}$ is high enough, then $k_{\text {eff }}^{2}$ has almost nothing to do with $d_{e} . k_{\text {eff }}^{2}$ rises with electrode thickness first and then descends with its rising, and the thickness corresponding to the maximum $k_{\text {eff }}^{2}$ is different with different electrode density and $v_{e}$, but they almost locate in the special area.
All above results indicate that the thickness, density, and acoustic velocity of electrode are very important, and they can be applied to optimize and design different kind of FBARs.

\section{Conflict of Interests}

The authors declare that there is no conflict of interests regarding the publication of this paper.

\section{Acknowledgments}

This work is supported by the National Natural Science Foundation of China (nos. 61201088, 11404257, and 11504291), Shaanxi Province Innovation Project for Science and Technology Overall Planning (no. 2012KTCL01-12), Industrialization Foundation of Shaanxi Educational Committee (no. 2011JG10), and Xi'an Technology Transferring and Promoting Project (CXY1342(5), CX1253(2)).

\section{References}

[1] J. D. Larson III, R. Ruby, P. Bradley, and Y. Oshmyansky, "BAW antenna duplex for the $1900 \mathrm{MHz}$ PCS band," in Proceedings of the IEEE Ultrasonics Symposium, pp. 887-890, Caesars Tahoe, NV, USA, October 1999.

[2] R. Ruby, P. Bradley, J. D. Larson III, and Y. Oshmyansky, "PCS $1900 \mathrm{MHz}$ duplexer using thin film bulk acoustic resonators (FBARs)," Electronics Letters, vol. 35, no. 10, pp. 794-796, 1999.

[3] R. C. Ruby, P. Bradley, Y. Oshmyansky, A. Chien, and J. D. Larson III, "Thin film bulk wave acoustic resonators (FBAR) for wireless applications," in Proceedings of the Ultrasonics Symposium, pp. 813-821, October 2001. 
[4] K. M. Lakin, J. Belsick, J. F. McDonald, and K. T. McCarron, "Improved bulk wave resonator coupling coefficient for wide bandwidth filters," in Proceedings of the IEEE Ultrasonics Symposium, vol. 1, pp. 827-831, IEEE, Atlanta, Ga, USA, October 2001.

[5] I. Voiculescu and A. N. Nordin, "Acoustic wave based MEMS devices for biosensing applications," Biosensors and Bioelectronics, vol. 33, no. 1, pp. 1-9, 2012.

[6] F. T. Chu, C. Li, Z. Z. Wang, and X. Z. Liu, "Aluminum Nitride Thin films on molybdenum/polyimide heterostructure for bulk acoustic resonators," Rare Metal Materials and Engineering, vol. 42, no. 10, pp. 2023-2026, 2013.

[7] A. J. Festschrift, J. K. Luo, Y. Q. Fu et al., "ZnO based SAW and FBAR devices for bio-sensing applications," Journal of NonNewtonian Fluid Mechanics, vol. 222, pp. 209-216, 2015.

[8] H. Zhang, G. Jiang, Z. Liu, S. Zhang, and L. Fan, "Optimizations of composited acoustic sensors with a microfluidic channel," Journal of Applied Physics, vol. 115, no. 13, Article ID 133502, 2014.

[9] A. Vorobiev and S. Gevorgian, "Intrinsically switchable thin film bulk acoustic wave resonators," Applied Physics Letters, vol. 104, no. 22, Article ID 222905, 2014.

[10] H. J. Lee, K. A. Hyun, and H. I. Jung, "A high-Q resonator using biocompatible materials at microwave frequencies," Applied Physics Letters, vol. 104, no. 2, Article ID 023509, 2014.

[11] M.-C. Chao, Z.-N. Huang, S.-Y. Pao, Z. Wang, and C. S. Lam, "Modified BVD-equivalent circuit of FBAR by taking electrodes into account," in Proceedings of the IEEE Ultrasonics Symposium, pp. 973-976, October 2002.

[12] Q. Chen and Q.-M. Wang, "The effective electromechanical coupling coefficient of piezoelectric thin-film resonators," Applied Physics Letters, vol. 86, no. 2, Article ID 022904, 2005.

[13] Y. Zhang, Z. Wang, and J. D. N. Cheeke, "Resonant spectrum method to characterize piezoelectric films in composite resonators," IEEE Transactions on Ultrasonics, Ferroelectrics, and Frequency Control, vol. 50, no. 3, pp. 321-333, 2003.

[14] T. Zhang, H. Zhang, Z.-Q. Wang, and S.-Y. Zhang, "Effects of electrodes on performance figures of thin film bulk acoustic resonators," Journal of the Acoustical Society of America, vol. 122, no. 3, pp. 1646-1651, 2007.

[15] N. F. Naumenko, "Advanced numerical technique for analysis of surface and bulk acoustic waves in resonators using periodic metal gratings," Journal of Applied Physics, vol. 116, no. 10, Article ID 104503, 2014.

[16] A. Kvasov and A. K. Tagantsev, "Thin film bulk acoustic wave resonators tuning from first principles," Journal of Applied Physics, vol. 113, no. 20, Article ID 204104, 2013.

[17] IEEE, "IEEE standard on piezoelectricity," Tech. Rep. 1761987, IEEE Transactions on Ultrasonics, Ferroelectrics and Frequency Control, 1996, 43:719. 

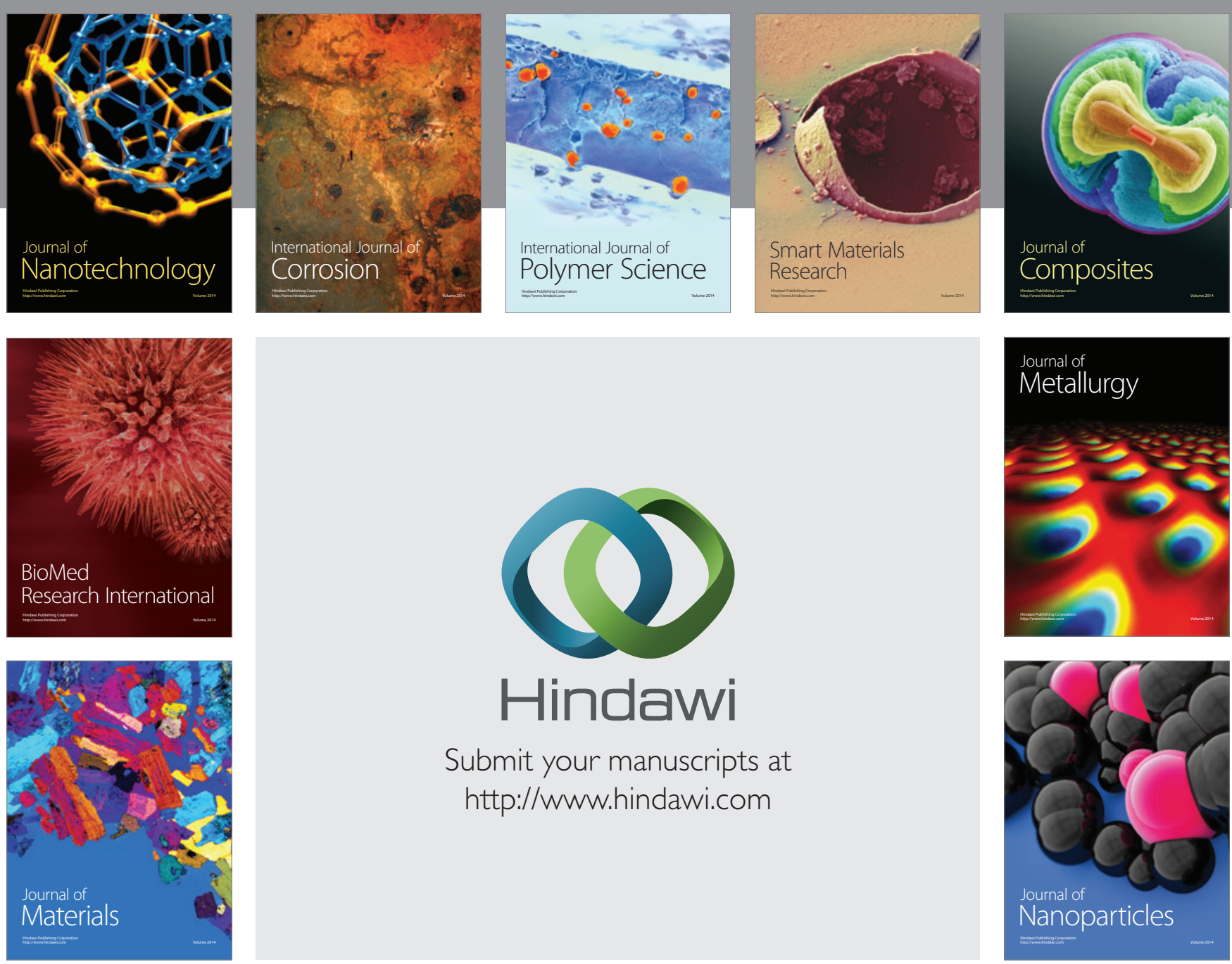

Submit your manuscripts at http://www.hindawi.com
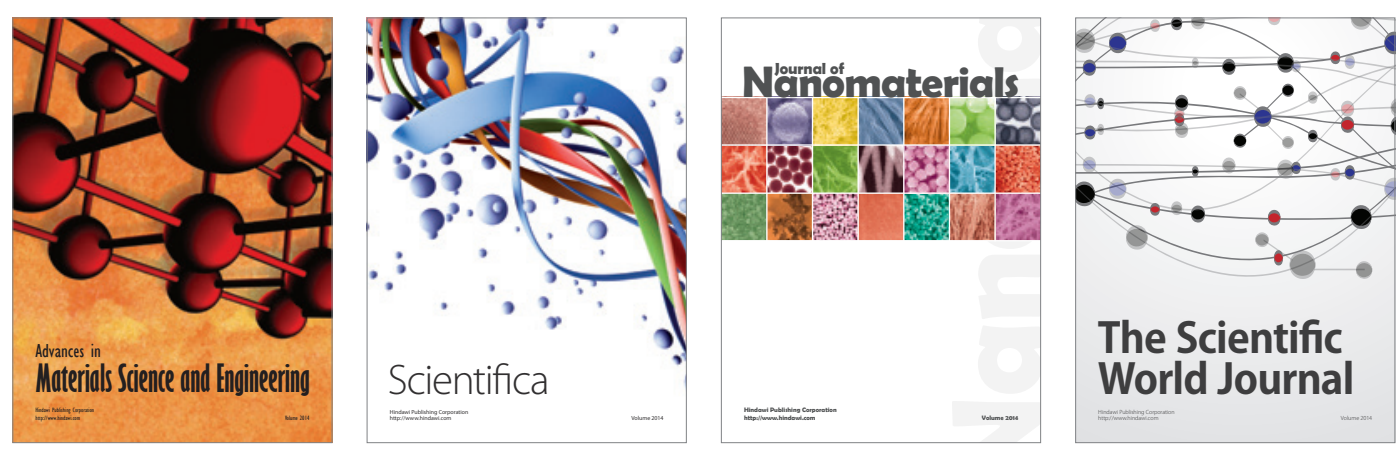

\section{The Scientific World Journal}
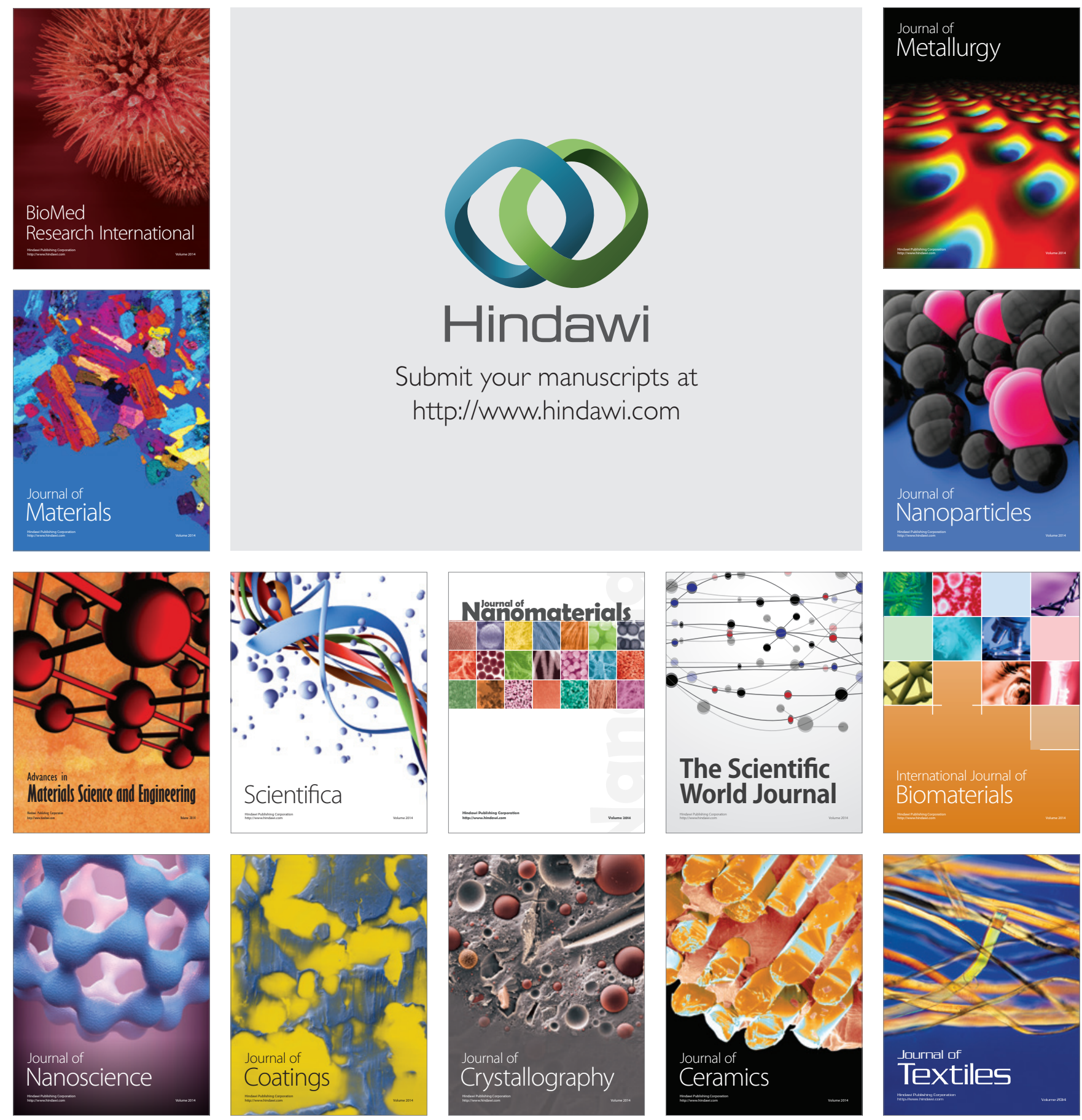